\title{
Cell stemness, epithelial-to-mesenchymal transition, and immunoevasion: Intertwined aspects in cancer metastasis
}

\author{
Simona Romano, Martina Tufano, Paolo D’Arrigo, Vincenza Vigorito, Salvatore Russo, \\ Maria Fiammetta Romano* \\ Department of Molecular Medicine and Medical Biotechnology, University of Naples "Federico II", Italy
}

\section{A R T I C L E I N F O}

\section{Keywords:}

Cancer stemness

Mesenchymal phenotype

Immunoevasion

PD-L1

\begin{abstract}
A B S T R A C T
Recent advances in tumor immunology, fostered by dramatic outcomes with cancer immunotherapy, have opened new scenarios in cancer metastasis. The cancer stemness/mesenchymal phenotype and an excess of immune suppressive signals are emerging as Intertwined aspects of human tumors. This review examines recent studies that explored the mechanistic links between cancer cell stemness and immunoevasion, and the evidence points to these key events in cancer metastasis as two sides of the same coin. This review also covers the mechanisms involved in tumor expression of programmed cell death ligand 1 (PD-L1), a major factor exploited by human neoplasias to suppress immune control. We highlight the convergence of mesenchymal traits and PDL1 expression and examine the functions of this immune inhibitory molecule, which confers cancer cell resistance and aggressiveness.
\end{abstract}

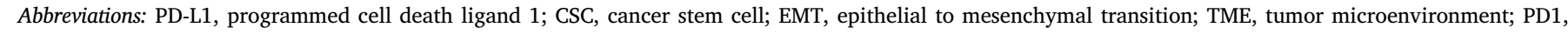

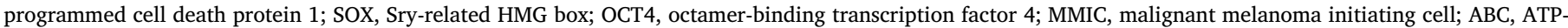

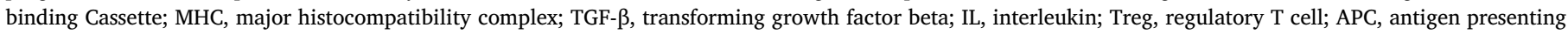

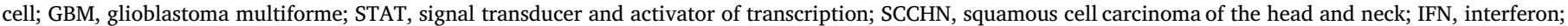

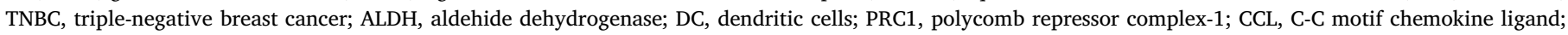

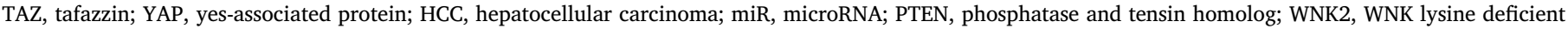

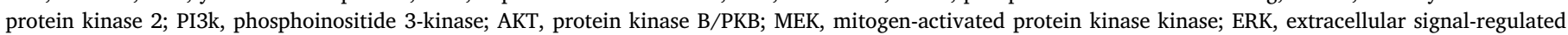

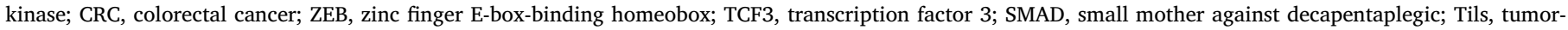

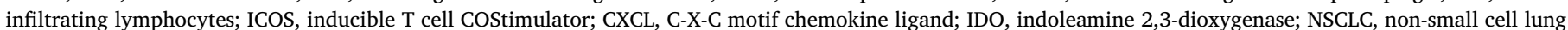

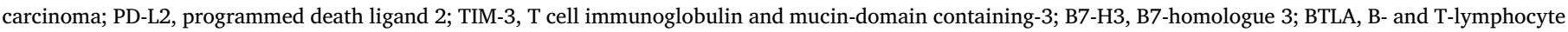

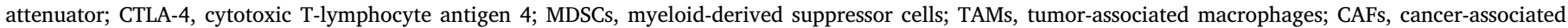

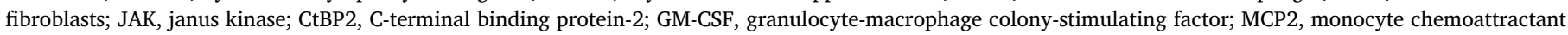

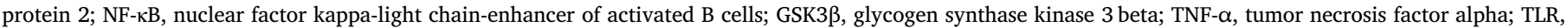

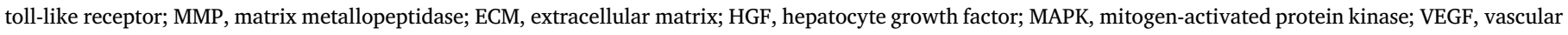

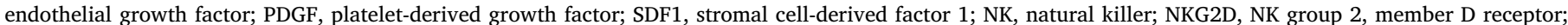

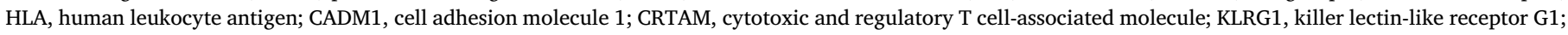

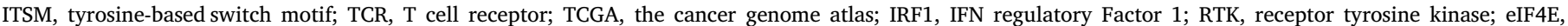

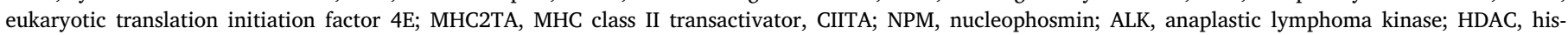

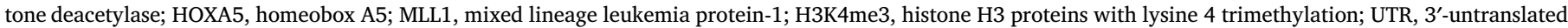

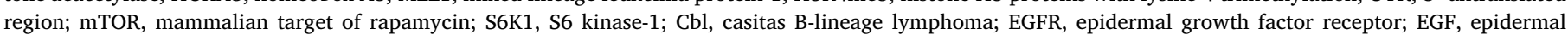

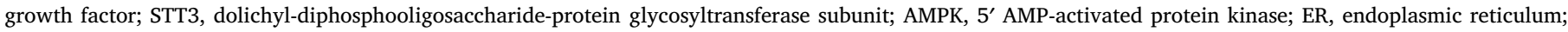

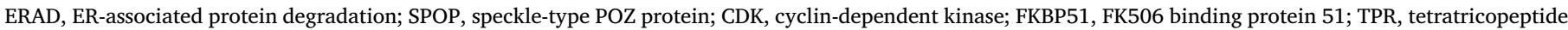

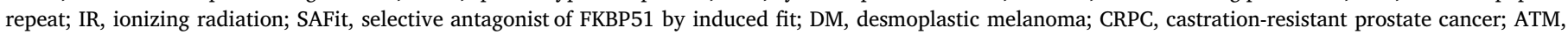
ataxia telengiectasia mutated kinase; SNHG20, small nucleolar RNA host gene 20; MUC1, mucin 1; TCF4, transcription factor 4; RPS6, ribosomal protein S6

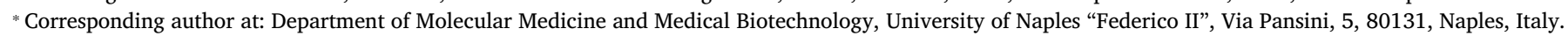

E-mail address: mariafiammetta.romano@unina.it (M.F. Romano).
} 


\section{Introduction}

In the last few decades, cancer research has put a lot of effort into understanding the mechanisms of metastasization. This research had led to the concept that disseminated metastasis arises from a small, heterogeneous fraction of cancer cells within the tumor, defined as CSCs [1]. When cancer cells undergo the EMT, they partially and transiently dedifferentiate. This transition provides an opportunity to adjust cellular gene expression and acquire a stem cell phenotype for self perpetuation and propagation [2]. Recent investigations on CSCs have revealed that these cells have a special immunoediting capacity, a process that leads the immune system to play supportive roles in tumorigenesis and metastasis. Many studies have investigated CSCs, which can be identified on the basis stemness markers. Those studies showed that CSCs could exploit multiple mechanisms to evade immune surveillance. In particular, the EMT state supports the tumor in acquiring immunoediting capacity and creating a permissive TME that promotes tumor growth and invasion. Due to its peculiar abilities to promote invasion and progression, but at the same time, to attract immune cells, EMT represents an important window for manipulating the immune system and reactivating it against cancer cells. Tumor immunoevasion is also supported by tumor expression of PD-L1. PD-L1 is among the best known and studied immune checkpoint co-inhibitory molecules. Antibody therapies directed against PD-L1 and its receptor, PD1, has dramatically changed the outcomes of many cancers that were considered incurable. This therapy has provided unprecedented results compared to chemotherapy.

Increasing evidence has supported the notion that PD-L1 has both immune regulatory effects and intrinsic supportive effects in tumor cells. Although PD-L1 expression in tumor cells is often acquired in an EMT context, genetic and epigenetic defects in tumor cells can lead to constitutive PD-L1 expression. In addition, PD-L1 expression in CSCs is directly supported by genes active in the embryo, like Sox2, Oct4, and members of the stemness-associated Wnt-signaling pathway. These studies have opened the door to new potential therapeutic agents that aim to counteract the function or expression of PD-L1, which would completely disarm CSCs. In this review, we examined studies that have addressed the immunoprivileged character of CSCs, either alone or in the context of EMT, which favors a permissive TME and supports the CSC phenotype. Moreover, we highlighted the reciprocal relationship between EMT and PD-L1, and at the same time, we investigated tumorspecific mechanisms that regulate the expression of PD-L1, independent of the TME.

\section{Cancer stem cells have intrinsic immunoprivileged properties}

CSCs comprise a small population of cells within the tumor, identifiable with a molecular marker or a marker combination. CSCs are characterized by the activation of genes related to early embryonic development that confer tumorigenic abilities, like self-renewal capacity, differentiation potential, and resistance to death. Studies from transplantation medicine have shown that physiologic stem cells have immunoprivileged properties [3-5]. The notion that CSCs might also have immunoevasive capabilities was inspired by the observation that host antitumor immunity could spare these cells. CSCs are indeed able to evade immune-mediated rejection in an immunocompetent host, even in the context of melanoma, which is highly immunogenic [6]. Schatton et al. studied MMICs, identified by the expression of the drug efflux transporter, ABC subfamily B, member 5 ABCB5. They demonstrated that MMICs could evade tumor immune surveillance more effectively than differentiated melanoma cells [7]. Several mechanisms in MMICs contribute to effector T cell tolerization, including: absent/low expression of tumor-associated antigens and of MHC class I molecules that preclude CD8 $\mathrm{T}$ cell activation, which prevents CD8 T cell-mediated killing; the expression of co-inhibitory signals upon $\mathrm{T}$ cell recognition; the secretion of immunosuppressive cytokines, like TGF- $\beta$ or
IL-10; the recruitment of Tregs; and the induction of Fas-mediated apoptotic $T$ cell death [6,7]. In addition, Schatton and Frank proposed that MMICs, like physiologic stem cells [8,9], might serve as functional APCs. Indeed, MMICs present tumoral antigens in the context of MHC class II molecules, either in the absence of positive co-stimulation or in the presence of a co-inhibitory immune ligand, like PD-L1 [6]. Consistently, Becker et al. showed that melanoma cells that expressed MHC class II molecules could induce clonal anergy in T cell clones, ex vivo [10].

The first immunobiological characterization of CSCs isolated from patients with GBM indicated that GBM-CSCs had lower immunogenicity and higher suppressive activity than non-CSC GBM cells [11]. Wei et al. isolated and generated single, CD133 cancer-initiating colonies from patients with GBM. They confirmed that glioma-cancer-initiating cells induced T cell suppression [12]. They also showed that STAT3 was constitutively active in GBM-CSCs. In turn, STAT3-induced tumor-secreted factors could activate STAT3 in immune cells of both the innate and acquired immune systems. This activation created a positive feedback loop between the tumor cells and the TME, which led to immunoevasion (for a comprehensive review of the role of STAT3 in immunosuppression, see Huynh et al. [13]).

Constitutive STAT3 activation was also found in $\mathrm{CD}_{4} 4^{+}$cells isolated from patients with SCCHN. CD44 is a well-characterized marker of a subpopulation of tumor cells associated with enhanced tumorigenesis, radioresistance, and chemoresistance [14]. In primary human SCCHN, constitutive STAT3 phosphorylation led to the selective expression of PD-L1 in CD44 ${ }^{+}$cells. In addition, CD $44^{+}$cells expressed higher levels of IFN $\gamma$ receptor 1 IFN $\gamma \mathrm{R} 1$, than $\mathrm{CD} 44^{-}$cells. Incubation of $\mathrm{CD}_{4} 4^{+}$cells with IFN $\gamma$ led to IFN $\gamma \mathrm{R} 1$ internalization and the phosphorylation of STAT1, a transcription factor that acts downstream of the IFN $\gamma$ R signaling. In turn, phosphorylated STAT1 upregulated PD-L1 expression. The increased sensitivity of $\mathrm{CD} 44^{+}$cells to IFN $\gamma$ suggested that SCCHN-CSCs could counteract lymphocyte attacks by enhancing their constitutive co-inhibitory immune receptor signaling more efficiently than their counterpart differentiated cells [14]. In lung carcinoma, CSCs that expressed the splicing variant of CD44 (CD44v) exhibited particularly high PD-L1 expression [15]. Also, TNBC cell lines, which exhibited stemness markers, like ALDH and CD44, showed increased PD-L1 levels, compared to their ALDH-negative and CD44 $4^{\text {low }}$ counterparts [16].

Treating TNBC cells with selective Wnt inhibitors or activators downregulated or upregulated PD-L1 expression, respectively, which implied that stemness-associated Wnt activity could engage in functional cross-talk with proteins involved in PD-L1 expression [16]. Indeed, TNBC-PD-L1 ${ }^{\text {high }}$ cells generated more mammospheres and showed increased in vivo tumor growth compared to TNBC-PD-L1 ${ }^{\text {low }}$ cells. Moreover, TNBC-PD-L1 ${ }^{\text {high }}$ cells established close contacts with $\mathrm{PD} 1^{+} \mathrm{T}$ cells in murine and human tumor samples; that finding supported the notion that CSCs were related to immune evasion [16]. In lung adenocarcinoma, Kerdidani et al. found that Wnt1 had paracrine effects on intratumoral DCs, including silencing the expression of chemokine genes. This mechanism tolerized intratumoral DCs to Wnt1 [17].

Immune evasion is a complex process that involves many signaling molecules. In double-negative prostate cancer, Su et al. found that PRC1 induced CD44 expression in cancer cells. In addition, PRC-1 induced the expression of the chemokine CCL2, which orchestrates the development of an immunosuppressive TME by recruiting M2-like tumorassociated macrophages and Tregs [18]. The TAZ and YAP kinases, which are downstream of the Hippo pathway, were found to act as oncogenes and transcriptional co-activators of stemness genes; in addition, they enhanced PD-L1 levels in breast and lung cancers [19]. A study on HCC showed that the stemness-related protein, Sox2, could directly bind to the PD-L1 promoter and transactivate its expression [20]. In cervical cancer, Dong et al. found that Oct4 transcriptionally regulated miR18a, which in turn, targeted PTEN, WNK2, and SOX6 


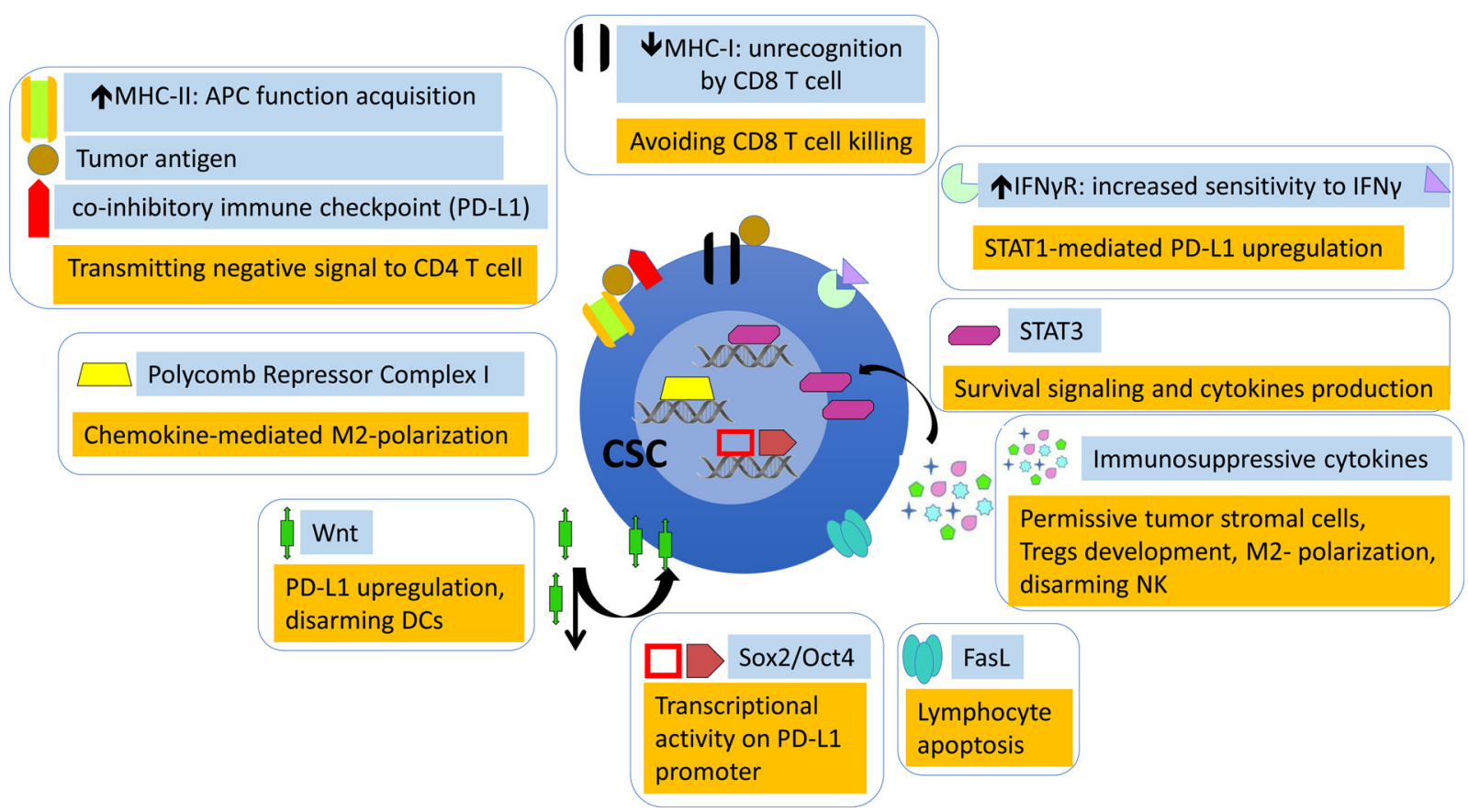

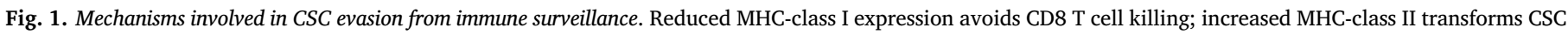

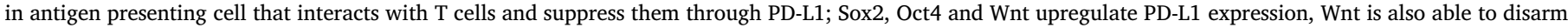

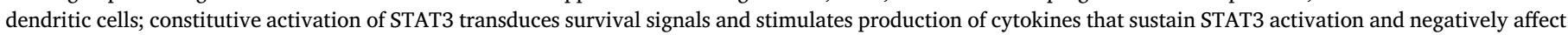

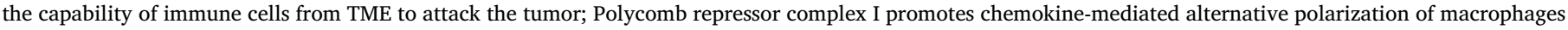
from TME; IFN $\gamma$ R renders CSC sensitive to signals from TME (i.e. IFN $\gamma$ ) to inducing PD-L1 expression; CSC-direct killing of immune cells through FasL.

genes. This mechanism inhibited p53 expression and activated PI3k/ Akt, MEK/Erk, and the Wnt/ $\beta$-catenin pathway, which ultimately led to PD-L1 upregulation [21]. CSCs purified from primary CRC showed weak immunogenicity, due to IL-4 production. Indeed, CRC-CSCs expressed both IL4 and its receptor. Blocking IL-4 activity stimulated T helper 1-mediated tumor-specific reactivity [22]. Fig. 1 illustrates the mechanisms exploited by CSCs to evade immune surveillance.

\section{The tumor microenvironment sustains immunoprivilege in CSCs}

Several lines of evidence support the notion that tumor cells interconvert from non-CSC to CSC phenotypes in the context of epigenetic changes associated with EMT (reviewed in [23]). CSC-enriched subpopulations in primary tumors have shown particularly prominent aspects of EMT-program activation [24]. A relatively small group of transcription factors orchestrate the genetic program that leads to EMT $[25,26]$. These gene expression changes are coordinated by three main families of transcription factors, namely, Snail (encoded by SNAI1 and SNAI2), ZEB proteins (ZEB1 and ZEB2), and basic helix-loop-helix proteins, TWIST1, TWIST2, and TCF3 [25-27]. Although a variety of stimuli have been implicated in the induction of EMT, multiple EMT models have shown that TGF- $\beta /$ Smad signaling plays a central role [28]. The role of TGF- $\beta$ in EMT was extensively reviewed previously $[27,28]$, and thus, we will not discuss it further in this review.

The TME is formed when cancer cells with a mesenchymal phenotype attract cells from the adaptive immune system and components of the innate immune system [29]. Indeed, EMT induces an adaptive immune response, characterized by a significant increase in CD3 Tils, Tregs, and several activation markers, including CD80, CD86, OX40 L, 4-1BB, ICOS, CD127, IFN $\gamma$, IFN $\gamma$-induced protein CXCL10, and an IFN $\gamma$ inducible enzyme IDO [29]. Moreover, in NSCLC, cells of the mesenchymal phenotype showed significantly higher levels of multiple immune checkpoint molecules, including PD-L1, PD-L2, PD1, TIM-3, B7-H3, BTLA, and CTLA-4, compared to cells of the epithelial phenotype [29].

The innate immune system includes MDSCs [30], TAMs [31], and CAFs [32]. These cells are particularly involved in the creation of a "niche", defined as an immunoprivileged, anatomically distinct TME that is devoted to supporting CSCs [33]. MDSCs are a heterogeneous subset of cells, from monocytic or granulocytic origin, which abundantly secrete IL- 6 and TGF- $\beta$; moreover, they recruit T helper 17 cells [34]. IL-6 supports cancer initiation and neoplastic progression through STAT3 signaling [35,36]. Moreover, IL-6 redirects the differentiation of myeloid progenitor cells to become MDSCs [35]. Through this mechanism, IL-6 interferes with the development of APCs that are necessary for priming cytotoxic $\mathrm{T}$ cells; this interference prevents $\mathrm{T}$ cell activation and induces $\mathrm{T}$ cell anergy or death. In addition to MDSCs, the primary sources of IL- 6 include the tumor cells, TAMs, $\mathrm{CD}^{+}{ }^{+} \mathrm{T}$ cells, and CAFs. In non-CSCs, IL- 6 regulates CSC-associated OCT4 gene expression through the IL-6-JAK1-STAT3 signal transduction pathway, as shown in breast cancer [36] and prostate cancer [32] settings. Giannoni et al. showed that, in a paracrine capacity, IL- 6 mediated the interplay between CAFs and prostate cancer cells, which led to an EMT-driven gain of CSC properties associated with aggressiveness and metastasis [32].

In ovarian cancer, a direct interaction between MDSCs and cancer cells triggered miR101 expression in the cancer cells. In turn, miR101 inhibited the co-repressor gene CtBP2, which increased the transcription of core cancer-stemness genes. A Kaplan Meier statistical analysis of overall survival confirmed that high MDSC density, high miR101 expression, and reduced CtBP2 expression were associated with a poor prognosis in patients with ovarian cancer [30].

TAMs derive from alternative macrophage polarization (M2) $[37,38]$. M2 macrophages cannot perform efficient antigen presentation or co-stimulation, and they attract Tregs, which participate in immunosuppression [39]. At the same time, TAMs sustain tumor-intrinsic cell signaling. In HCC, TAMs secreted IL-8, which promoted the EMT program through the JAK/STAT3/SNAIL signaling pathway [40]. Su et al. demonstrated a positive feedback loop between EMT and TAMs, 
fostered by the GM-CSF secreted by breast cancer cells [41]. GM-CSF activated TAMs, which in turn, secreted CCL8, also known as MCP2. CCL8 ultimately activated EMT through NF-kB-mediated stimulation of the axis formed by PI3k/Akt/GSK3 $\beta /$ Snail $[42,43]$. TAMs also secrete TNF- $\alpha$, which plays an EMT-promoting role in breast cancer, mediated by SNAIL upregulation [44]. In pancreatic cancer, TAM-secreted IL-10 is a central promoter of EMT [45]. When pancreatic cancer cells were cocultured with immune suppressive M2-polarized macrophages, the presence of IL-10 induced aggressive behavior. In particular, IL-10 induced EMT by activating TLR4, MMP2 and MMP9 [46], which worked in concert with the transcription factor, Snail [45].

CAFs play an important pathogenic role in HCC. In more than $80 \%$ of cases, HCC develops within the context of cirrhosis. Due to chronic inflammation, HCC is accompanied by an enrichment of activated fibroblasts. Compared to normal tissue fibroblasts, these CAFs proliferate at a higher rate and show enhanced extracellular matrix production. Moreover, it has been shown that CAFs secrete HGF, which activates the tyrosine kinase receptor, c-MET, in HCC cells [47]. After ligand binding, c-MET is phosphorylated at two tyrosine residues [47]. Then, HGF/MET activates oncogenic signaling pathways, namely Ras, PI3k, MAPK/STAT, and $\beta$-catenin/Wnt, which lead to cancer stemness [47]. CAFs also secrete a number of other growth factors, including VEGF, HGF, and PDGF [48]. However, CAFs mostly contribute to immunoevasion by producing SDF1 (also known as CXCL12), which is involved in M2-macrophage polarization [49].

\section{EMT serves as a window for NK cells to control tumor progression}

The EMT process is tightly connected to inflammation [50]. Ricciardi et al. investigated an inflammatory-induced EMT model with different cancer cell types. They proposed that, during EMT, cancer cells acquired some immune-modulatory mechanisms shared by mesenchymal stromal cells, but they also emphasized that cancer cells could perform some peculiar tumor-specific immunomodulatory properties. That study found a number of immune-modulatory mechanisms, mostly dependent on the cancer cell type, that could account for EMTinduced immune suppression. Specifically, an impairment in NK or T cell function and an expansion of both Tregs and B cells could hamper the host immune attack against the tumor. The suppression mechanism of immune cells was partly mediated by activating the IDO pathway [51]. Alternatively, NK cells can positively influence EMT, as shown by Huergo-Zapigo et al [52]. Those authors found that NK cells produced proteomic changes in melanoma cells that largely overlapped with those of cytokine-induced EMT. This NK-mediated tumor-cell editing depended on the activation of NKG2D and the natural killer cytotoxicity receptor, NKp30, in addition to the release of TNFa and IFN $\gamma$. In turn, IFN $\gamma$ upregulated IDO and increased HLA-I surface expression, which conferred resistance to NK-mediated killing. Furthermore, NK-induced IFN $\gamma$ secretion might induce autophagy, which protects tumor cells against both NK- and T cell attacks [51].

As suggested by studies from Lopez-Soto et al. and Chockley et al., EMT is an important window for the immune system to control tumor progression. In this view, manipulating NK cells during this window could effectively redirect the immune cell subset against the tumor $[53,54]$. Indeed, Lopez-Soto et al. found that, in CRC, the acquisition of mesenchymal characteristics induced the expression of ligands for some NK receptors, particularly the NKG2D receptor, which rendered tumor cells in the EMT state more susceptible to elimination by NK cells [53] Similarly, a study by Chockley et al. demonstrated that, during EMT, the induction of CADM1 regulated a metastasis-specific immunosurveillance, mediated by NK cells. More specifically, when CADM1 bound to the CRTAM receptor on NK cells, NK cytotoxicity was activated [54]. NK activity was also enhanced when, during EMT, Ecadherin downmodulation prevented the adhesion molecule engagement and inhibition of KLRG1 [54]. Overall, these studies supported the concept that the modality of integrating NK receptor signaling could determine whether NK cells became activated. Manipulating these signaling receptors during EMT could promote the innate ability of NK cells to detect and kill cancer cells, while sparing normal cells.

\section{Mesenchymal tumors have the highest PD-L1 expression levels}

PD-L1 (CD274, B7-H1) is a transmembrane protein, mainly expressed by cells from the adaptive immune system, whose function is to limit the clonal expansion of antigen-specific $\mathrm{CD} 8^{+}$cytotoxic $\mathrm{T}$ cells and $\mathrm{CD}^{+}{ }^{+} \mathrm{T}$ helper cells [55]. PD-L1 binds to the inhibitory checkpoint receptor, PD1, which is expressed by activated $\mathrm{T}$ lymphocytes. This binding transmits an inhibitory signal, mediated by the de-phosphorylation of ITSM on the TCR. A recent study performed multi-platform analyses of the transcriptome, methylome, and transcription-factor binding sites with datasets from TCGA and an innovative, one-class logistic regression, machine-learning algorithm [56]. Their findings emphasized the notion that, among various tumor types, mesenchymal tumors had the highest PD-L1 expression levels and the largest leukocyte fractions among the different cancer types [57]. As previously discussed, the tumoral EMT-phenotype attracts immune cells, which could explain the acquired nature of tumor-PD-L1 expression that characterizes the tumor EMT state [29]. A thorough study of melanoma [58] showed that PD-L1 was mainly induced and regulated by IFN $\gamma$ and the type II IFN receptor signaling pathway. This signaling pathway included JAK1 and JAK2, several STATs, and other modulators, and it ultimately led to the binding of IRF1 to the PD-L1 promoter. IFN $\gamma$ treatment resulted in a clear, reliable pattern, where the induction of the JAK2/STAT1/IRF1 axis led to PD-L1 upregulation [58], which is typical of the type II IFN canonical pathway. IL- 6 also played a role in the stimulation of PD-L1 expression by upregulating STAT3 via an autocrine/paracrine pathway [59]. Xu et al. highlighted the control of PDL1 translation in HCC, which was mediated by oncogene expression concomitant with EMT [60]. The authors found that MYC, in cooperation with RTK/RAS signaling, markedly increased PD-L1 expression and promoted tumor formation, EMT, and metastasis [60]. In the context of both MYC and KRAS activation (but not KRAS activation alone), the ribosome footprints changed for a subset of transcripts. More precisely, MYC upregulated the translation of several proteins, including cytokines involved in immune cell attraction and inflammation, factors necessary for cell cycle progression and cell motility, and PD-L1 [60]. When the phosphorylation of eIF4E was inhibited by the eFT508 compound, PD-L1 and several other translational targets were significantly and selectively downregulated [60]. Cerezo et al. found that eIF4F regulated the translation of mRNA that encoded the transcription factor, STAT1 [61]. Through this mechanism, eIF4F could control IFN- $\gamma$-induced PD-L1 expression in cancer cells [61]. Chen et al. found that, in cancer cells, the EMT-related gene, ZEB1, acted in concert with the stemness-related gene, Oct4, in increasing PD-L1 expression [20]. Deng et al. proposed that FOXO transcription factors, which played contrasting roles in Myc and Wnt/ $\beta$-catenin oncogenic pathways, could downregulate tumor PD-L1 expression, and thus, promote antitumor immunity [62].

\section{Other mechanisms of aberrant PD-L1 expression in human tumors}

In cold (non-inflamed) tumors, aberrant PD-L1 expression can occur with genomic alterations. For example, elevated copy numbers of chromosome 9p24 (where the CD274 gene is located) have been found in many human cancers, including small-cell lung cancer [63], squamous cell carcinoma of the oral cavity [64], cervical cancer [65], ovarian cancer [66], breast cancer [67], melanoma, bladder cancer, sarcomas, and prostate cancer [68]. In lymphoid tumors, recurrent rearrangements involving MHC2TA, induced the overexpression of CD274 (PD-L1 gene) and CD273 (PD-L2 gene) and the downregulation 
Table 1

Mechanisms that regulate tumor PD-L1 expression. All the mechanisms, but one, increase protein level.

\begin{tabular}{|c|c|c|c|}
\hline Causal factor & Mechanism & Regulation & Ref \\
\hline \multirow[t]{5}{*}{ Genomic alterations } & Increased copy number of 9p24 & Transcription & {$[63,64,65,66,67,68]$} \\
\hline & MHC2TA rearrangements & Transcription & [69] \\
\hline & $\begin{array}{l}\text { Structural variations at chromosome 9p24.1 } \\
\text { induce }\end{array}$ & Post-transcription & [78] \\
\hline & $\begin{array}{c}\text { PD-L1 3'-UTR disruption } \\
\text { and CD274 }\end{array}$ & & \\
\hline & mRNA stabilization & & \\
\hline \multirow[t]{6}{*}{ Epigenetic alterations } & $\downarrow$ HDAC8 activity & Transcription & {$[71,77]$} \\
\hline & $\downarrow$ Promoter-methylation & & \\
\hline & $\begin{array}{l}\text { Promoter H3K4-3methylation } \\
\text { by MLL1 }\end{array}$ & Transcription & {$[72]$} \\
\hline & $\downarrow \mathrm{miR}-200$ & Post-transcription & {$[104,108]$} \\
\hline & $\begin{array}{l}\text { /activation of } \\
\text { ZEB/EMT }\end{array}$ & & \\
\hline & $\begin{array}{l}\text { defect in miR-34a, } \\
\text { miR-424, } \\
\text { miR-138-5p, } \\
\text { miR-142-5p }\end{array}$ & Post-transcription & {$[73,76]$} \\
\hline $\mathrm{IFN} \gamma$ & JAK2/STAT1/IRF1 & Transcription & {$[58,61]$} \\
\hline Mutated Ras & RAS/Erk & Post-transcription & {$[60,79,80,105]$} \\
\hline MYC & PI3k/Akt & Translation & \\
\hline \multicolumn{4}{|l|}{ Mutated PTEN } \\
\hline NPM/ALK & STAT3 & Transcription & {$[70]$} \\
\hline EGF & JAK/STAT3 & Transcription & {$[58,59,82]$} \\
\hline IL6 & PI3k/Akt & Translation & \\
\hline EGF & GSK3 $\beta$ inactivation & Post-translational, membrane transport and stabilization & {$[84,85,87]$} \\
\hline \multirow[t]{2}{*}{ CDK4/CDK6 } & SPOP phosporylation & Post-translational & [87] \\
\hline & & PD-L1 degradation & \\
\hline $\begin{array}{l}\text { STT3 } \\
\text { FKBP51s }\end{array}$ & PD-L1 glycosylation & $\begin{array}{l}\text { Post-translational } \\
\text { protein maturation }\end{array}$ & {$[83,88]$} \\
\hline
\end{tabular}

of surface HLA II expression [69]. The oncogenic, fusion protein, NPM/ ALK, was shown to upregulate PD-L1 expression in neoplastic T lymphoma cells via STAT3 activation [70].

PD-L1 expression can also be regulated epigenetically. In melanoma cells, the class I histone deacetylase, HDAC8, acts on a complex formed by HOXA5 and STAT3 to inhibit PD-L1 expression [71]. In pancreatic tumor cells, Lu et al. highlighted another form of epigenetic PD-L1 regulation [72]. They showed that increasing expression and activity of the histone lysine methyltransferase MLL1 generated a cluster of H3K4me3, which attached to the CD274 promoter region that flanks the PD-L1 transcription start site. Inhibiting MLL1 reduced the level of H3K4me3 clusters on the CD274 promoter region and downregulated PD-L1 expression. That result suggested that MLL1 played a relevant role in PD-L1 expression in pancreatic cancer [72]. In addition, several miRs have been implicated in regulating PD-L1 expression [73-76]. MiR binding to the 3 'UTR of PD-L1 mRNAs hampered translation and reduced PD-L1 production [73-76]. During anti-PD1 therapy for patients with NSCLC, the $P D-L 1$ promoter was methylated, which reduced PD-L1 expression [77]. Aberrant PD-L1 expression might also occur when PD-L1 mRNAs are stabilized by the disruption of its $3^{\prime}$-UTR region, which occurs in various tumors [78].

Disruptions in cellular signaling can also cause aberrant PD-L1 expression. For example, the loss of PTEN, which occurs in many human cancers, promotes PD-L1 translation by activating the PI3k/Akt/ mTOR/S6K1 pathway $[79,80]$. This pathway facilitates the entrance of PD-L1 transcripts into polysomes, which increases PD-L1 translation, as shown in human gliomas $[77,78]$. In NSCLC, the ubiquitin ligases, Cbl-b and c-Cbl, were also involved in regulating PD-L1 expression [81]. By inactivating STAT3/Akt/Erk signaling, Cbl-b and c-Cbl inhibited PD-L1 expression. Accordingly, the levels of Cbl-b and c-Cbl expression were negatively correlated with the level of PD-L1 expression in wild-type NSCLC tissues that overexpressed EGFR [81]. In NSCLC, Okita et al. also highlighted a role for EGF in PD-L1 expression, via the PI3K/Akt and JAK/STAT pathways [82]. Hsu et al. showed that the two isoforms of the N-glycosyltransferase, STT3 (isoforms A and B), played a pivotal role in PD-L1 glycosylation [83]. The STT3 N-glycosyltransferases are located in the endoplasmic-reticulum of mammalian cells. Both STT3 isoforms are particularly expressed during EMT [83]. The STT3 proteins mediate Nglycosylation of PD-L1, which stabilizes PD-L1 by protecting the protein from ubiquitination and proteasomal degradation. Specifically, glycosylation antagonizes the binding of GSK3 $\beta$, which interacts with PD-L1 to induce its proteasomal degradation. PD-L1 degradation also depends on phosphorylation, which is mediated by the betatransducin repeat containing E3 ubiquitin protein ligase $\beta$-TrCP [84]. GSK3 $\beta$ can be inactivated by EGF, which stabilizes PD-L1. Inhibition of EGF by gefitinib affected PD-L1 levels by increasing its degradation [84]. Similarly, in colon cancer, Chen and coworkers found that EGF facilitated PD-L1 transport to the surface of cancer cells, which stabilized the protein [85]. They also found that insulin had a stimulatory effect on PD-L1 synthesis, which was mediated by activating the PI3k/ Akt pathway. The synergy between EGF and insulin effects induced a remarkable increase in PD-L1 expression on the surfaces of CSCs. This elevated expression enhanced the ability of CSCs to form spheres [85]. The importance of proper glycosylation for maintaining PD-L1 stability was also emphasized by Cha et al [86]. They showed that AMPK-induced phosphorylation of PD-L1 at serine 195 could alter PD-L1 glycosylation. This specific phosphorylation could critically determine PDL1 accumulation in the ER, and its subsequent degradation by the ERAD pathway [86]. Zhang et al. found that the cullin 3-SPOP was an E3 ligase that mediated proteasomal degradation of PD-L1. Cyclin D-CDK4 could phosphorylate SPOP, which then promoted PD-L1 degradation [87]. Consequently, loss-of-function SPOP mutations prevented ubiquitination-mediated PD-L1 degradation and accounted for increased PD-L1 levels in primary human prostate cancer specimens [87].

A recent study found that the short isoform of the isomerase, immunophilin FKBP51 (FKBP51 s), could assist in PD-L1 maturation [88]. Alternative FKBP5 splicing removes the TPR domain of FKBP5, which confers an important function, namely it is capable of accessing the ER to promote PD-L1 maturation [88]. Biochemical analyses of glioma cell lines showed that naïve PD-L1 was complexed with FKBP51 s in the ER, 
but the glycosylated PD-L1 was found in the Golgi apparatus [88]. A knockdown of FKBP51 s severely reduced the level of glycosylated PDL1, whether it was constitutively expressed or ionizing radiation-induced [88]. The essential role of isomerase activity in PD-L1 protein maturation was confirmed by selectively inhibiting the catalytic function of FKBP51 with SAFit2 [89]; this inhibition produced results similar to those observed with FKBP51 s silencing [88]. Table 1 summarizes the mechanisms regulating tumor PD-L1 expression.

\section{PD-L1 sustains resistance to cell death and feeds the CSC/EMT activation loop}

The concept that PD-L1 could exert intrinsic oncogenic functions, independent of immune suppression [90], dates back to 2007, when Azuma et al. demonstrated that PD-L1 transduced an anti-apoptotic signal in cancer cells that led to a resistance to killing by cytotoxic $\mathrm{T}$ lymphocytes and apoptosis induced by Fas or drugs. The integrity of the intracellular PD-L1 domain was essential for regulating the resistance signal [91]. In detail, the conserved RMLDVEKC motif is responsible for counteracting cell death, and the DTSSK motif acts as a negative regulator of this PD-L1 function [92]. Interestingly, a variety of human carcinomas carry somatic mutations that affect residues within the inhibitory DTSSK motif.

The engagement of PD-L1 with its cognate receptor, PD1, on T cells reinforced the pro-survival function of PD-L1 [92]. In breast cancer [93] and pancreatic carcinoma [94], tumor-immune cell interactions, through PD-L1/PD1, stimulated Akt and Erk phosphorylation in cancer cells. Conversely, PD-L1 targeting negatively regulated the PI3k/Akt/ mTOR signaling pathway, upregulated PTEN expression, and reduced MMP2 and MMP9 levels, in pancreatic cancer [94]. In tyrosine kinaseresistant NSCLC models, targeting or disrupting mTORC2 abrogated the morphological and functional traits of EMT and, at the same time, impaired PDL1 expression [95]. In melanoma and ovarian cancer, PDL1 downmodulation resulted in reduced phosphorylation of the mTORC1 substrate, P70S6K, which drove the cells into a slowly proliferating cancer cell phenotype. In addition, as a consequence of PD-L1 downmodulation, the anti-proliferative effect of rapamycin was enhanced [95]. When PD-L1 was knocked out, glioma growth was counteracted, both in vitro and in vivo [96].

Qiu et al. identified a physical interaction between PD-L1 and HRas, which led to Ras/Erk activation. In glioma cells, this activation promoted EMT, migration, and invasion [96]. Xue et al. studied glioma specimens and found a correlation between PD-L1 expression and malignancy features, like the expression of proangiogenic VEGF and the proliferative marker Ki-67 [97]. When PD-L1 was silenced in melanoma cells, Clark et al. showed reduced metastasis in an in vivo mouse model [98]. In DM, a rare subtype of melanoma with frequent local recurrences, PD-L1 played a role in promoting cell proliferation [99]. DM is a unique form of melanoma, characterized by a prominent host response and high PD-L1 expression. Those authors found a positive correlation between PD-L1 and p53 expression, the Ki-67 proliferation index, tumoral and stromal CD8 $\mathrm{T}$ cell infiltration, and stromal Treg infiltration. They also found that PD-L1 expression in cancer was correlated with tumor thickness, mitosis, recurrence, and metastasis [99].

In CRPC, ATM is an apical activator of the DNA damage response [100]. This kinase showed significantly enhanced expression in prostate cancer tissues from patients with CRPC, and high ATM levels were associated with EMT features and high PD-L1 levels [100]. An ATM knockout in CRPC cells resulted in reduced PD-L1 levels. The same effect on PD-L1 was obtained when cells were treated with a JAK inhibitor; this treatment resulted in a significant reduction in the expression of EMT-associated genes [100]. These findings suggested that the ATM/JAK/PD-L1 axis played a role in the transition from hormone dependence to hormone independence in prostate cancer, and also in the progression of CRPC cells to metastasis [100]. The relevance of the ATM/JAK/PD-L1 axis in the induction of EMT was further assessed in esophageal squamous cell carcinoma. The ATM/JAK pathway was activated by a small nucleolar RNA, termed SNHG20, which was widely upregulated in cancer tissues and cell lines. Accordingly, SNHG20 levels were positively correlated with tumor size, lymph node metastasis, TNM stage, and tumor grade [101].

In lung adenocarcinoma, PD-L1 expression was significantly elevated in patient tissues that had a prevailing EMT phenotype, which was characterized by increases in SNAI1 and VIM expression [102]. The role that the EMT inducer, ZEB1, played in PD-L1 regulation was assessed in esophageal carcinoma [103]. When small, interfering RNAs were used to mediate a ZEB1 knockdown, PD-L1 expression and EMT features were suppressed [103]. In squamous cell lung cancer, Chen et al. found that ZEB1 upregulated PD-L1 expression through the suppression of miR200 [104]. In breast cancer, Alsuliman et al. clearly showed a bi-directional interplay between EMT status and PD-L1 expression [105]. Most PD-L1-positive cell lines had mesenchymal features, in contrast to PD-L1-negative cell lines, which demonstrated luminal differentiation characteristics. When PD-L1 was upregulated in breast cancer cells, a typical mesenchymal phenotype was induced, characterized by the upregulation of CD44/vimentin and the downregulation of CD24/E-cadherin. In PTEN-mutated breast cancer cells, EMT features and PD-L1 expression appeared to depend on constitutive activation of the PI3k/Akt pathway. In breast cancer cell lines with a functional PTEN, significant correlations were found between ZEB2, SNAI1, ZEB1, and PD-L1. In TNBC, the claudin-low phenotype showed a high EMT score, which was associated with a high degree of immune cell infiltrate, high PD-L1 expression, and a poor prognosis. Targeting PD-L1 resulted in the attenuation of EMT features in this aggressive breast cancer subtype [105].

Maeda et al. found that MUC1, a heterodimeric protein that is overexpressed in approximately $90 \%$ of TNBCs, functioned as an oncoprotein [106]. MUC1 acts as a node for integrating multiple signaling pathways at the cell membrane and in the nucleus. MUC1 activates multiple cell signaling pathways, including PI3k/Akt, MEK/Erk, $\beta$-catenin/TCF4/Myc, and NF-кB pathways. These autoinductive circuits increased PD-L1 transcription. An increase in PD-L1 transcription was also mediated by downmodulating miR-34a and miR-200c, two direct suppressors of PD-L1 [106].

PD-L1 could positively regulate the expression of stemness genes, like OCT-4, NANOG, and SOX2 [107-109]. PD-L1 also promoted tumorinitiating cell generation in several cancers, including melanoma, ovarian cancer [107], breast cancer [108] and cervical carcinoma [109]. The strict interplay between PD-L1 and the hallmarks of tumor malignancy is schematized in Fig. 2.

Although few in number, some findings conflicted with the role of PD-L1 in tumor progression. For example, in one study, PD-L1-silencing in cholangiocarcinoma cells enhanced their tumorigenicity [110]. In that study, patients with low tumor PD-L1 expression had a worse prognosis than those with high PD-L1 expression [110]. Those findings suggested that the PD-L1 immune checkpoint could inhibit cancer stemness in some specific tumoral contexts. In another study, CDK4 and CDK6 kinases destabilized PD-L1, which led to PD-L1 downregulation in cancer cells [87]. In particular, cyclin D-CDK4-mediated phosphorylation of SPOP induced PD-L1 degradation via ubiquitination [87]. Conversely, CDK4/6 inhibition increased PD-L1 levels and reduced the number of Tils in mouse tumors and in primary human prostate cancer [87]. Curiously, in bladder cancer, Jinesh et al. found that epithelial cells that expressed PD-L1 in the membrane also co-expressed E-cadherin and CD24, a glycoprotein expressed in differentiating neuroblasts. These epithelial cells rapidly formed tumors outside the primary TME in nude mice, which indicated that these cells possessed features of immune evasion, increased stemness, and malignancy. Conversely, mesenchymal cells exhibited constitutive TGF- $\beta$ signaling and were less tumorigenic than these epithelial cells [111]. 


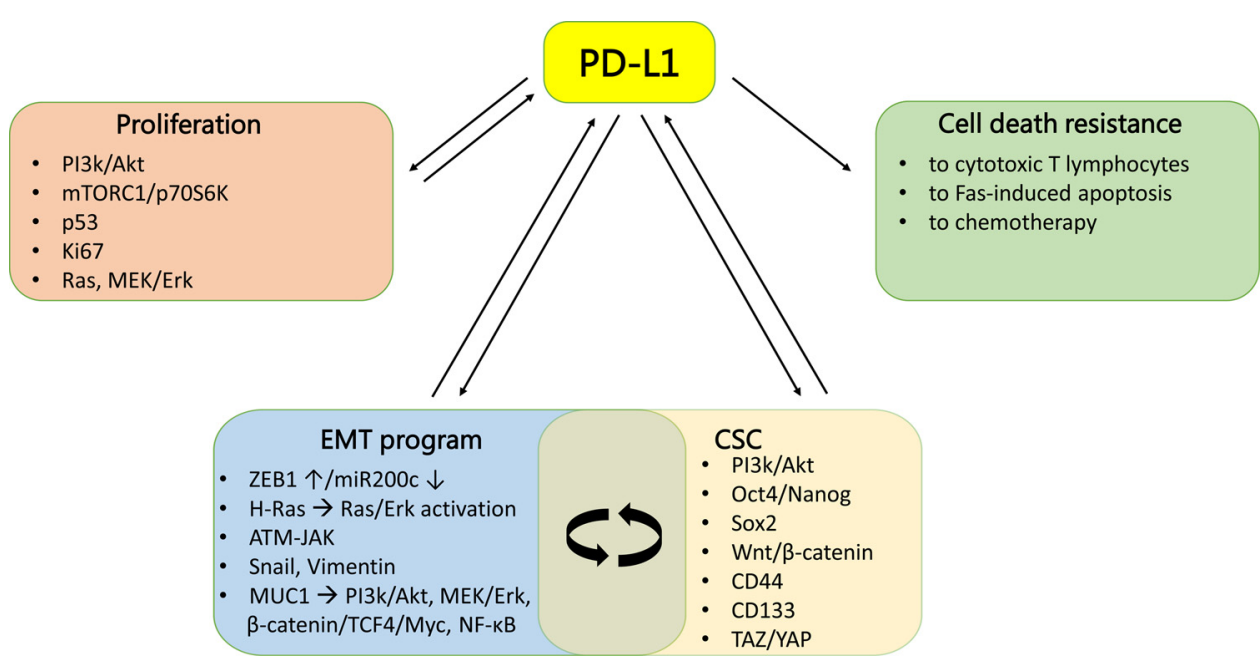

Fig. 2. Reciprocal regulation between $P D-L 1$ and hallmarks of tumor-malignancy. PD-L1 expression increases by activation of proliferationrelated signaling pathways. In turn, PD-L1 sustains activation of such tumor growth-promoting signaling pathways. A similar bidirectionality is registered between EMT/CSC-related genes and pathways that induce PD-L1 expression; pathways related to cancer renewal and invasion are, indeed, positively affected by PD-L1. Finally, PD-L1 supports resistance to cell death induced by both lymphocyte killing and chemotherapy.

\section{Intrinsic expression of PD1 in many tumor types}

Recent studies have evaluated cancer transcriptomic and proteomic data. Murine and human melanomas were shown to include cancer subpopulations that expressed PD1, even in mice that lacked adaptive immunity [112]. Indeed, targeting the PD1 pathway exerted anti-melanoma tumor growth effects in mice with fully intact immunity, in mice that were severely immunocompromised, and in hosts that were deficient in T cells, B cells, and innate immune cells [112]. Interestingly, PD1 was not found in all the melanoma cells present in heterogeneous tumor samples. Rather, PD1 expression was restricted to small melanoma subpopulations that were, nonetheless, critically important for tumor growth.

PD1 can be activated via paracrine interactions with the PD-L1 ligand or via autocrine signaling that interacts with PD1 in tumor cells. In turn, PD1 activation in melanoma cells increased the phosphorylation of RPS6, an effector of mTOR signaling [112]. Melanoma PD1dependent RPS6 phosphorylation could be reversed with specific mTOR inhibition, but not with PI3k inhibition. This result demonstrated that PD1 receptors that resided on the surface of melanoma cells could activate downstream mTOR signaling, independent of PI3k activation, to promote tumor proliferation [112].

In HCC cell lines and clinical HCC tissues, PD1 was expressed in subpopulations. In those cells, PD1 intrinsically promoted tumor progression, in the absence of an immunological environment [113]. Specifically, the cytosolic domains of PD1 were found to interact with both eiF4E and RPS6. This interaction promoted the phosphorylation of mTOR effector proteins [113]. Interestingly, PD1 activation augmented p-70S6k levels in melanoma cells and enhanced tumor growth; in contrast, PD1 dampened mTOR signaling in T cells, which led to diminished T cell proliferation [114]. Conversely, PD1 appeared to play a distinct role in lung cancer, where a blockade of PD1 promoted tumor cell proliferation [115].

The triggers of PD1 expression in tumor cells are currently unknown, but it has been speculated that multiple factors may be involved, such as gene copy number alterations, epigenetic factors, microenvironmental signals, etc [115]. The existence of tumor cellintrinsic PD1 expression and its tumor-regulatory effects might explain why a PD1 blockade displayed efficacy in patients with less immunogenic features; why no response was detected to immunotherapies that targeted other immune checkpoints; and/or why the clinical activity and safety profile of anti-PD1 therapy were superior to those of anti-CTLA-4 therapy [115].

\section{Conclusion}

Two major aspects of CSC plasticity are responsiveness to microenvironmental cues and in vivo quiescence. CSCs mediate tumor metastasis by maintaining the plasticity necessary to transition between epithelial and mesenchymal states. The stroma surrounding tumors and the immune system modulate cancer plasticity and release signals, like IFN $\gamma$ and IL6, that induce tumor PD-L1 expression. These signals, in addition to other suppressive signals, either tumor-intrinsic or generated by TME-EMT cross-talk, confer an inherent immunoprivileged condition. A plethora of studies have supported the concept that tumor PD-L1 expression is particularly high during activation of the EMT genetic program. Moreover, many studies have shown that mesenchymal tumors have a high potential susceptibility to checkpoint inhibitors. In turn, signals generated through PD-L1 can sustain the EMT genetic program. The activation of the immune system has proven to be an important resource in combating human neoplasias, in addition to the classic pillars of surgery, chemotherapy, and radiotherapy. Interventions that rely on immune checkpoint inhibitors, such as antiPD1/PD-L1 immunotherapies, have indeed provided impressive outcomes for many tumors that are considered incurable. However, a caveat has emerged with the finding that PD-L1 expression in a tumor does not necessarily mean that anti-PD1/PD-L1 immunotherapy will be successful [90]. The complex, dynamic interactions between tumors and the TME foster immunoevasion circuits that support intrinsic or acquired resistance to anti-PD1/PD-L1 treatments. However, according to the recent advances on PD-L1, even in cases of resistance, where reactivating the immune system would require targeting immunecheckpoints different from PD-L1/PD1, a combined treatment approach that includes disarming PD-L1-mediated tumor resistance could aid immune cells in killing cancer cells. PD-L1 transduces pro-tumoral signals that are reasonably self-maintained by the tumor; for example, tumors can express more than one type of receptor for PD-L1, apart from PD1 [112, https://www.proteinatlas.org/ENSG00000121594CD80/pathology]. Disarming PD-L1 can also be a successful strategy in cold tumors, where a number of genetic and epigenetic mechanisms support PD-L1 expression. In these cases, immunotherapy is often preceded by treatment with oncolytic viruses, to favor tumor-infiltrating lymphocytes. In the future, the creation of innovative tools that hamper PD-L1 expression/function, can enrich the therapeutic armamentarium for fighting cancer. CSCs represent the leading cause of cancer therapy failure. The increasing evidence that a CSC/EMT/PD-L1 axis exists suggests that developing innovative tools for disarming PDL1 might open up new possibilities for eradicating cancer. 


\section{Declaration of Competing Interest}

The authors declare that there are no conflicts of interest.

\section{Acknowledgements}

We thank the Regione Campania SATIN grant 2018-2020; the STAR L1-Junior Principal Investigator Grants 2018 (funded by Unina and CSP) and the Cardiovascular Service S.r.l.

\section{References}

[1] N. Ahmed, R. Escalona, D. Leung, E. Chan, G. Kannourakis, Tumour microenvironment and metabolic plasticity in cancer and cancer stem cells: perspectives on metabolic and immune regulatory signatures in chemoresistant ovarian cancer stem cells, Semin. Cancer Biol. 53 (2018) 265-281, https://doi.org/10.1016/j. semcancer.2018.10.002

[2] H. Wang, J.J. Unternaehrer, Epithelial-mesenchymal transition and cancer stem cells: at the crossroads of differentiation and dedifferentiation, Dev. Dyn. 248 (2019) 10-20, https://doi.org/10.1002/dvdy.24678.

[3] L. Gallacher, L. Li, M.L. Baroja, A. Majumdar, K. Chadwick, A. Rouleau, et al., Human embryonic stem cells possess immune-privileged properties, Stem Cells 22 (2004) 448-456, https://doi.org/10.1634/stemcells.22-4-448.

[4] M. Drukker, H. Katchman, G. Katz, S. Even-Tov Friedman, E. Shezen, E. Hornstein, O. Mandelboim, et al., Human embryonic stem cells and their differentiated derivatives are less susceptible to immune rejection than adult cells, Stem Cells 24 (2006) 221-229, https://doi.org/10.1634/stemcells.2005-0188.

[5] C.A. Koch, P. Geraldes, J.L. Platt, Immunosuppression by embryonic stem cells, Stem Cells 26 (2008) 89-98, https://doi.org/10.1634/stemcells.2007-0151.

[6] T. Schatton, M.H. Frank, Antitumor immunity and cancer stem cells, Ann. N. Y. Acad. Sci. 1176 (2009) 154-169, https://doi.org/10.1111/j.1749-6632.2009. 04568.x.

[7] T. Schatton, U. Schütte, N.Y. Frank, Q. Zhan, A. Hoerning, S.C. Robles, et al., Modulation of T-cell activation by malignant melanoma initiating cells, Cancer Res. 15 (70) (2010) 697-708, https://doi.org/10.1158/0008-5472.CAN-09-1592.

[8] C.J. Chang, M.L. Yen, Y.C. Chen, C.C. Chien, H.I. Huang, C.H. Bai, et al., Placentaderived multipotent cells exhibit immunosuppressive properties that are enhanced in the presence of interferon-gamma, Stem Cells 24 (2006) 2466-2477, https:// doi.org/10.1634/stemcells.2006-0071.

[9] R. Romieu-Mourez, M. François, M.N. Boivin, J. Stagg, J. Galipeau, Regulation of MHC class II expression and antigen processing in murine and humanmesenchymal stromal cells by IFN-gamma, TGF-beta, and cell density, J. Immunol. 179 (2007) 1549-1558, https://doi.org/10.4049/jimmunol.179.3.1549.

[10] J.C. Becker, T. Brabletz, C. Czerny, C. Termeer, E.B. Bröcker, Tumor escape mechanisms from immunosurveillance: induction of unresponsiveness in a specific MHC-restricted CD4 + human T cell clone by the autologous MHC class II + melanoma, Int. Immunol. 5 (1993) 1501-1508, https://doi.org/10.1093/intimm/ 5.12 .1501$.

[11] T. Di Tomaso, S. Mazzoleni, E. Wang, G. Sovena, D. Clavenna, A. Franzin, et al., Immunobiological characterization of cancer stem cells isolated from glioblastoma patients, Clin. Cancer Res. 1 (16) (2010) 800-813, https://doi.org/10.1158/10780432.CCR-09-2730.

[12] J. Barr, J. Wei, L.Y. Kong, Y. Wang, A. Wu, A.K. Sharma, et al., Glioblastoma cancer-initiating cells inhibit T-cell proliferation and effector responses by the signal transducers and activators of transcription 3 pathway, Mol. Cancer Ther. 9 (2010) 67-78, https://doi.org/10.1158/1535-7163.MCT-09-0734.

[13] J. Huynh, N. Etemadi, F. Hollande, M. Ernst, M. Buchert, The JAK/STAT3 axis: a comprehensive drug target for solid malignancies, Semin. Cancer Biol. 45 (2017) 13-22, https://doi.org/10.1016/j.semcancer.2017.06.001.

[14] Y. Lee, J.H. Shin, M. Longmire, H. Wang, H.E. Kohrt, H.Y. Chang, et al., CD44 + cells in head and neck squamous cell carcinoma suppress T-cell-mediated immunity by selective constitutive and inducible expression of PD-L1, Clin. Cancer Res. 22 (2016) 3571-3581, https://doi.org/10.1158/1078-0432.CCR-15-2665.

[15] M. Ozaki, M. Nishino, A.E. Hegab, J. Hamamoto, S. Kagawa, D. Arai, et al., Variant CD44 expression is enriching for a cell population with cancer stem cell-like characteristics in human lung adenocarcinoma, J. Cancer 8 (2017) 1774-1785, https://doi.org/10.7150/jca.19732.

[16] L. Castagnoli, V. Cancila, S.L. Cordoba-Romero, S. Faraci, G. Talarico, B. Belmonte, et al., WNT signaling modulates PD-L1 expression in the stem cell compartment of triple-negative breast cancer, Oncogene (2019), https://doi.org/10.1038/s41388019-0700-2.

[17] D. Kerdidani, P. Chouvardas, A.R. Arjo, I. Giopanou, G. Ntaliarda, Y.A. Guo, et al., Wnt1 silences chemokine genes in dendritic cells and induces adaptive immune resistance in lung adenocarcinoma, Nat. Commun. 10 (2019) 1405, https://doi. org/10.1038/s41467-019-09370-Z.

[18] W. Su, H.H. Han, Y. Wang, B. Zhang, B. Zhou, Y. Cheng, et al., The polycomb repressor complex 1 drives double-negative prostate cancer metastasis by coordinating stemness and immune suppression, Cancer Cell (19) (2019) S1535-6108, https://doi.org/10.1016/j.ccell.2019.06.009 pii:30300-9.

[19] H.J. Janse van Rensburg, T. Azad, M. Ling, Y. Hao, B. Snetsinger, P. Khanal, et al., The hippo pathway component TAZ promotes immune evasion in human cancer through PD-L1, Cancer Res. 78 (2018) 1457-1470, https://doi.org/10.1158/
0008-5472.CAN-17-3139.

[20] T. Chen, J. Du, G. Lu, Cell growth arrest and apoptosis induced by Oct4 or Nanog knockdown in mouse embryonic stem cells: a possible role of Trp53, Mol. Biol. Rep. 39 (2012) 1855-1861, https://doi.org/10.1007/s11033-011-0928-6.

[21] M.A. Mamun, K. Mannoor, J. Cao, F. Qadri, X. Song, SOX2 in cancer stemness: tumor malignancy and therapeutic potentials, J. Mol. Cell Biol. (2018), https:// doi.org/10.1093/jmcb/mjy080.

[22] A. Volonté, T. Di Tomaso, M. Spinelli, M. Todaro, F. Sanvito, L. Albarello, et al., Cancer-initiating cells from colorectal cancer patients escape from T cell-mediated immunosurveillance in vitro through membrane-bound IL-4, J. Immunol. 192 (2014) 523-532, https://doi.org/10.4049/jimmunol.1301342.

[23] U.E. Martinez-Outschoorn, M. Peiris-Pagés, R.G. Pestell, F. Sotgia, M.P. Lisanti, Cancer metabolism: a therapeutic perspective, Nat. Rev. Clin. Oncol. 14 (2017) 11-31, https://doi.org/10.1038/nrclinonc.2016.60.

[24] T. Shibue, R.A. Weinberg, EMT, CSCs, and drug resistance: the mechanistic link and clinical implications, Nat. Rev. Clin. Oncol. 14 (2017) 611-629, https://doi. org/10.1038/nrclinonc.2017.44.

[25] B. De Craene, G. Berx, Regulatory networks defining EMT during cancer initiation and progression, Nat. Rev. Cancer 13 (2013) 97-110, https://doi.org/10.1038/ nrc3447.

[26] I. Muqbil, J. Wu, A. Aboukameel, R.M. Mohammad, A.S. Azmi, Snail nuclear transport: the gateways regulating epithelial-to-mesenchymal transition? Semin. Cancer Biol. 27 (2014) 39-45, https://doi.org/10.1016/j.semcancer.2014.06.003.

[27] P. Simeone, M. Trerotola, J. Franck, T. Cardon, M. Marchisio, I. Fournier, et al., The multiverse nature of epithelial to mesenchymal transition, Semin. Cancer Biol. 16 (November) (2018), https://doi.org/10.1016/j.semcancer.2018.11.004 pii: S1044-579X(18)30086-5.

[28] A. Moustakas, C.H. Heldin, Induction of epithelial-mesenchymal transition by transforming growth factor $\beta$, Semin. Cancer Biol. 22 (2012) 446-454, https://doi org/10.1016/j.semcancer.2012.04.002.

[29] Y. Lou, L. Diao, E.R. Cuentas, W.L. Denning, L. Chen, Y.H. Fan, et al., Epithelialmesenchymal transition is associated with a distinct tumor microenvironment including elevation of inflammatory signals and multiple immune checkpoints in lung adenocarcinoma, Clin. Cancer Res. 22 (2016) 3630-3642, https://doi.org/ 10.1158/1078-0432.CCR-15-1434.

[30] T.X. Cui, I. Kryczek, L. Zhao, E. Zhao, R. Kuick, M.H. Roh, et al., Myeloid-derived suppressor cells enhance stemness of cancer cells by inducing microRNA101 and suppressing the corepressor CtBP2, Immunity 39 (2013) 611-621, https://doi org/10.1016/j.immuni.2013.08.025.

[31] H. Lu, K.R. Clauser, W.L. Tam, J. Fröse, X. Ye, E.N. Eaton, et al., A breast cancer stem cell niche supported by juxtacrine signalling from monocytes and macrophages, Nat. Cell Biol. 16 (2014) 1105-1117, https://doi.org/10.1038/ncb3041.

[32] E. Giannoni, F. Bianchini, L. Masieri, S. Serni, E. Torre, L. Calorini, et al., Reciprocal activation of prostate cancer cells and cancer-associated fibroblasts stimulates epithelial-mesenchymal transition and cancer stemness, Cancer Res. 70 (2010) 6945-6956, https://doi.org/10.1158/0008-5472.CAN-10-0785.

[33] J. Ye, D. Wu, P. Wu, Z. Chen, J. Huang, The cancer stem cell niche: cross talk between cancer stem cells and their microenvironment, Tumour Biol. 35 (2014) 3945-3951, https://doi.org/10.1007/s13277-013-1561-x.

[34] T. Kitamura, B.-Z. Qian, J.W. Pollard, Immune cell promotion of metastasis, Nat Rev. Immunol. 15 (2015) 73-86, https://doi.org/10.1038/nri3789.

[35] D.T. Fisher, M.M. Appenheimer, S.S. Evans, The two faces of IL-6 in the tumor microenvironment, Semin. Immunol. 26 (2014) 38-47, https://doi.org/10.1016/j smim.2014.01.008.

[36] S.Y. Kim, J.W. Kang, X. Song, B.K. Kim, Y.D. Yoo, Y.T. Kwon, et al., Role of the IL 6-JAK1-STAT3-Oct-4 pathway in the conversion of non-stem cancer cells into cancer stem-like cells, Cell. Signal. 25 (2013) 961-969, https://doi.org/10.1016/j. cellsig.2013.01.007.

[37] T. Rőszer, Understanding the misterious M2 macrophage through activation markers and effector mechanisms, Mediators Inflamm. (2015) 1-16, https://doi. org/10.1155/2015/816460.

[38] E. Grimaldi, P. Carandente, F. Scopacasa, M.F. Romano, M. Pellegrino, R. Bisogni, et al., Evaluation of the monocyte counting by two automated haematology analysers compared with flow cytometry, Clin. Lab. Haematol. 25 (2005) 91-97, https://doi.org/10.1111/j.1365-2257.2005.00676.x.

[39] V. Plaks, N. Kong, Z. Werb, The cancer stem cell niche: how essential is the niche in regulating stemness of tumor cells? Cell Stem Cell 16 (2015) 225-238, https:// doi.org/10.1016/j.stem.2015.02.015.

[40] X.T. Fu, Z. Dai, K. Song, Z.J. Zhang, Z.J. Zhou, S.L. Zhou, et al., Macrophagesecreted IL-8 induces epithelial-mesenchymal transition in hepatocellular carcinoma cells by activating the JAK2/STAT3/Snail pathway, Int. J. Oncol. 46 (2015) 587-596, https://doi.org/10.3892/ijo.2014.2761.

[41] S. Su, Q. Liu, J. Chen, J. Chen, F. Chen, C. He, et al., A positive feedback loop between mesenchymal-like cancer cells and macrophages is essential to breast cancer metastasis, Cancer Cell 25 (2014) 605-620, https://doi.org/10.1016/j.ccr. 2014.03.021.

[42] B. Zhang, C. Yin, H. Li, L. Shi, N. Liu, Y. Sun, et al., Nir1 promotes invasion of breast cancer cells by binding to chemokine (C-C motif) ligand 18 through the PI3K/Akt/GSK3ß/Snail signalling pathway, Eur. J. Cancer 49 (2013) 3900-3913, https://doi.org/10.1016/j.ejca.2013.07.146.

[43] S. Julien, I. Puig, E. Caretti, J. Bonaventure, L. Nelles, F. van Roy, et al., Activation of NF-kappaB by Akt upregulates Snail expression and induces epithelium mesenchyme transition, Oncogene 26 (2007) 7445-7456, https://doi.org/10.1038/ sj.onc. 1210546

[44] C. Zhou, A.M. Nitschke, W. Xiong, Q. Zhang, Y. Tang, M. Bloch, et al., Proteomic analysis of tumor necrosis factor-alpha resistant human breast cancer cells reveals 
a MEK5/Erk5-mediated epithelial-mesenchymal transition phenotype, Breast Cancer Res. 10 (2008) R105, https://doi.org/10.1186/bcr2210.

[45] C.Y. Liu, J.Y. Xu, X.Y. Shi, W. Huang, T.Y. Ruan, P. Xie, et al., M2-polarized tumorassociated macrophages promoted epithelial-mesenchymal transition in pancreatic cancer cells, partially through TLR4/IL-10 signaling pathway, Lab. Invest. 93 (2013) 844-854, https://doi.org/10.1038/labinvest.2013.69.

[46] S. Asuthkar, A.K. Nalla, C.S. Gondi, D.H. Dinh, M. Gujrati, S. Mohanam, et al., Gadd45a sensitizes medulloblastoma cells to irradiation and suppresses MMP-9mediated EMT, Neuro Oncol. 13 (2011) 1059-1073, https://doi.org/10.1093/ neuonc/nor109.

[47] E.Y. Lau, J. Lo, B.Y. Cheng, M.K. Ma, J.M. Lee, J.K. Ng, et al., Cancer-associated fibroblasts regulate tumor-initiating cell plasticity in hepatocellular carcinoma through c-Met/FRA1/HEY1 signaling, Cell Rep. 15 (2016) 1175-1189, https:// doi.org/10.1016/j.celrep.2016.04.019.

[48] M.R. Junttila, F.J. de Sauvage, Influence of tumour microenvironment heterogeneity on therapeutic response, Nature 501 (2013) 346-354, https://doi.org/10. 1038/nature12626.

[49] L. Sánchez-Martín, A. Estecha, R. Samaniego, S. Sánchez-Ramón, M. Vega, P. Sánchez-Mateos, The chemokine CXCL12 regulates monocyte-macrophage differentiation and RUNX3 expression, Blood 117 (2011) 88-97, https://doi.org/10. 1182/blood-2009-12-258186.

[50] A. Sica, A. Mantovani, P. Allavena, F. Balkwill, Cancer-related inflammation, Nature 454 (2008) 436-444, https://doi.org/10.1038/nature07205.

[51] M. Zanotto, M. Ricciardi, G. Malpeli, G. Bassi, O. Perbellini, M. Chilosi, et al., Epithelial-to-mesenchymal transition (EMT) induced by inflammatory priming elicits mesenchymal stromal cell-like immune-modulatory properties in cancer cells, Br. J. Cancer 112 (2015) 1067-1075, https://doi.org/10.1038/bjc.2015.29.

[52] L. Huergo-Zapico, M. Parodi, C. Cantoni, C. Lavarello, J.L. Fernández-Martínez, A. Petretto, et al., NK-cell editing mediates epithelial-to-mesenchymal transition via phenotypic and proteomic changes in melanoma cell lines, Cancer Res. 78 (2018) 3913-3925, https://doi.org/10.1158/0008-5472.CAN-17-1891.

[53] A. Astudillo, A. López-Soto, L. Huergo-Zapico, J.A. Galván, L. Rodrigo, A.G. de Herreros, et al., Epithelial-mesenchymal transition induces an antitumor immune response mediated by NKG2D receptor, J. Immunol. 190 (2013) 4408-4419, https://doi.org/10.4049/jimmunol.1202950.

[54] P.J. Chockley, J. Chen, G. Chen, D.G. Beer, T.J. Standiford, V.G. Keshamouni, Epithelial-mesenchymal transition leads to NK cell-mediated metastasis-specific immunosurveillance in lung cancer, J. Clin. Invest. 128 (2018) 1384-1396, https://doi.org/10.1172/JCI97611.

[55] P. D'Arrigo, M. Tufano, A. Rea, V. Vigorito, N. Novizio, S. Russo, et al., Manipulation of the immune system for cancer defeat: a focus on the $\mathrm{T}$ cell inhibitory checkpoint molecules, Curr. Med. Chem. (2018), https://doi.org/10. 2174/0929867325666181106114421.

[56] A. Sokolov, E.O. Paull, J.M. Stuart, One-class detection of cell states in tumor subtypes, Pac. Symp. Biocomput. 21 (2016) 405-416, https://doi.org/10.1142/ 9789814749411_0037.

[57] T.M. Malta, A. Sokolov, A.J. Gentles, T. Burzykowski, L. Poisson, J.N. Weinstein, et al., Machine learning identifies stemness features associated with oncogenic dedifferentiation, Cell 173 (2018) 338-354, https://doi.org/10.1016/j.cell.2018. 03.034 e15.

[58] A. Garcia-Diaz, D.S. Shin, B.H. Moreno, J. Saco, H. Escuin-Ordinas, G.A. Rodriguez, et al., Interferon receptor signaling pathways regulating PD-L1 and PD-L2 expression, Cell Rep. 19 (2017) 1189-1201, https://doi.org/10.1016/j. celrep.2017.04.031.

[59] Y.H. Jin, W. Hou, H.S. Kang, C.S. Koh, B.S. Kim, The role of interleukin-6 in the expression of PD-1 and PDL-1 on central nervous system cells following infection with Theiler's murine encephalomyelitis virus, J. Virol. 87 (2013) 11538-11551, https://doi.org/10.1128/JVI.01967-13.

[60] Y. Xu, M. Poggio, H.Y. Jin, Z. Shi, C.M. Forester, Y. Wang, et al., Translation control of the immune checkpoint in cancer and its therapeutic targeting, Nat. Med. 25 (2019) 301-311, https://doi.org/10.1038/s41591-018-0321-2.

[61] M. Cerezo, R. Guemiri, S. Druillennec, I. Girault, H. Malka-Mahieu, S. Shen, et al., Translational control of tumor immune escape via the eIF4F-STAT1-PD-L1 axis in melanoma, Nat. Med. 24 (2018) 1877-1886, https://doi.org/10.1038/s41591 018-0217-1.

[62] Y. Deng, F. Wang, T. Hughes, J. Yu, FOXOs in cancer immunity: knowns and unknowns, Semin. Cancer Biol. 50 (2018) 53-64, https://doi.org/10.1016/j. semcancer.2018.01.005.

[63] J. George, M. Saito, K. Tsuta, R. Iwakawa, K. Shaishi, A.H. Scheel, et al., Genomic amplification of CD274 (PD-L1) in small-cell lung cancer, Clin. Cancer Res. 23 (2017) 1220-1226, https://doi.org/10.1158/1078-0432.CCR-16-1069.

[64] M. Straub, E. Drecoll, N. Pfarr, W. Weichert, R. Langer, A. Hapfelmeier, et al., CD274/PD-L1 gene amplification and PD-L1 protein expression are common events in squamous cell carcinoma of the oral cavity, Oncotarget 7 (2016) 12024-12034, https://doi.org/10.18632/oncotarget.7593.

[65] B.E. Howitt, H.H. Sun, M.G. Roemer, A. Kelley, B. Chapuy, E. Aviki, et al., Genetic basis for PD-L1 expression in squamous cell carcinomas of the cervix and vulva, JAMA Oncol. 2 (2016) 518-522, https://doi.org/10.1001/jamaoncol.2015.6326.

[66] J. Budczies, C. Denkert, B. Győrffy, P. Schirmacher, A. Stenzinger, Chromosome 9p copy number gains involving PD-L1 are associated with a specific proliferation and immune-modulating gene expression program active across major cancer types, BMC Med. Genomics 10 (2017) 74, https://doi.org/10.1186/s12920-017-0308-8.

[67] M.T. Barrett, K.S. Anderson, E. Lenkiewicz, M. Andreozzi, H.E. Cunliffe, C.L. Klassen, et al., Genomic amplification of 9p24.1 targeting JAK2, PD-L1, and PD-L2 is enriched in high-risk triple negative breast cancer, Oncotarget 6 (2015) 26483-26493, https://doi.org/10.18632/oncotarget.4494.
[68] Cancer Genome Atlas Research Network, Comprehensive molecular characterization of gastric adenocarcinoma, Nature 513 (2014) 202-209, https://doi.org/ 10.1038/nature13480.

[69] C. Steidl, S.P. Shah, B.W. Woolcock, L. Rui, M. Kawahara, P. Farinha, et al., MHC class II transactivator CIITA is a recurrent gene fusion partner in lymphoid cancers, Nature 471 (2011) 377-381, https://doi.org/10.1038/nature09754.

[70] M. Paessler, M. Marzec, Q. Zhang, A. Goradia, P.N. Raghunath, X. Liu, et al., Oncogenic kinase NPM/ALK induces through STAT3 expression of immunosuppressive protein CD274 (PD-L1, B7-H1), PNAS 105 (2008) 20852-20857, https://doi.org/10.1073/pnas.0810958105.

[71] Y.F. Wang, F. Liu, S. Sherwin, M. Farrelly, X.G. Yan, A. Croft, et al., Cooperativity of HOXA5 and STAT3 Is Critical for HDAC8 Inhibition-Mediated Transcriptional Activation of PD-L1 in Human Melanoma Cells, J. Invest. Dermatol. 138 (2018) 922-932, https://doi.org/10.1016/j.jid.2017.11.009.

[72] C. Lu, A.V. Paschall, H. Shi, N. Savage, J.L. Waller, M.E. Sabbatini, et al., The MLL1-H3K4me3 axis-mediated PD-L1 expression and pancreatic cancer immune evasion, J. Natl. Cancer Inst. 109 (2017) djw283, , https://doi.org/10.1093/jnci/ djw283.

[73] S. Xu, Z. Tao, B. Hai, H. Liang, Y. Shi, T. Wang, et al., miR-424(322) reverses chemoresistance via T-cell immune response activation by blocking the PD-L1 immune checkpoint, Nat. Commun. 7 (2016) 11406, https://doi.org/10.1038/ ncomms11406.

[74] X. Wang, J. Li, K. Dong, F. Lin, M. Long, Y. Ouyang, et al., Tumor suppressor miR34a targets PD-L1 and functions as a potential immunotherapeutic target in acute myeloid leukemia, Cell. Signal. 27 (2015) 443-452, https://doi.org/10.1016/j. cellsig.2014.12.003.

[75] L. Zhao, H. Yu, S. Yi, X. Peng, P. Su, Z. Xiao, et al., The tumor suppressor miR-1385p targets PD-L1 in colorectal cancer, Oncotarget 7 (2016) 45370-45384, https:// doi.org/10.18632/oncotarget.9659.

[76] L. Jia, Q. Xi, H. Wang, Z. Zhang, H. Liu, Y. Cheng, et al., miR-142-5p regulates tumor cell PD-L1 expression and enhances anti-tumor immunity, Biochem. Biophys. Res. Commun. 488 (2017) 425-431, https://doi.org/10.1016/j.bbrc. 2017.05.074.

[77] Y. Duan, Y. Zhang, C. Xiang, Y. Wang, C. Liu, Y. Zhang, PD-L1 promoter methylation mediates the resistance response to anti-PD-1 therapy in NSCLC patients with EGFR-TKI resistance, Oncotarget 8 (2017) 101535-101544, https://doi.org/ 10.18632/oncotarget.21328.

[78] K. Kataoka, Y. Shiraishi, Y. Takeda, S. Sakata, M. Matsumoto, S. Nagano, et al., Aberrant PD-L1 expression through 3'-UTR disruption in multiple cancers, Nature 534 (2016) 402-406, https://doi.org/10.1038/nature18294.

[79] A.T. Parsa, J.S. Waldron, A. Panner, C.A. Crane, I.F. Parney, J.J. Barry, et al., Loss of tumor suppressor PTEN function increases B7-H1 expression and immunoresistance in glioma, Nat. Med. 13 (2007) 84-88, https://doi.org/10.1038/ nm1517.

[80] C.A. Crane, A. Panner, J.C. Murray, S.P. Wilson, H. Xu, L. Chen, et al., PI (3) kinase is associated with a mechanism of immunoresistance in breast and prostate cancer, Oncogene 28 (2009) 306-312, https://doi.org/10.1038/onc.2008.384.

[81] S. Wang, L. Xu, X. Che, C. Li, L. Xu, K. Hou, et al., E3 ubiquitin ligases Cbl-b and cCbl downregulate PD-L1 in EGFR wild-type non-small cell lung cancer, FEBS Lett. 59 (2018) 621-630, https://doi.org/10.1002/1873-3468.12985.

[82] R. Okita, A. Maeda, K. Shimizu, Y. Nojima, S. Saisho, M. Nakata, PD-L1 overexpression is partially regulated by EGFR/HER2 signaling and associated with poor prognosis in patients with non-small-cell lung cancer, Cancer Immunol. Immunother. 66 (2017) 865-876, https://doi.org/10.1007/s00262-017-1986-y.

[83] J.M. Hsu, C.W. Li, Y.J. Lai, M.C. Hung, Posttranslational modifications of PD-L1 and their applications in cancer therapy, Cancer Res. 78 (2018) 6349-6353, https://doi.org/10.1158/0008-5472.CAN-18-1892.

[84] C.-W. Li, S.-O. Lim, W. Xia, H.-H. Lee, L.-C. Chan, C.-W. Kuo, et al., Glycosylation and stabilization of programmed death ligand-1 suppresses T-cell activity, Nat. Commun. 7 (2016) 12632, https://doi.org/10.1038/ncomms12632.

[85] M. Chen, A. Sharma, Y. Lin, Y. Wu, Q. He, Y. Gu, et al., Insluin and epithelial growth factor (EGF) promote programmed death ligand 1 (PD-L1) production and transport in colon cancer stem cells, BMC Cancer 19 (2019) 153, https://doi.org/ 10.1186/s12885-019-5364-3

[86] J.H. Cha, W.H. Yang, W. Xia, Y. Wei, L.C. Chan, S.O. Lim, et al., Metformin promotes antitumor immunity via endoplasmic-reticulum-associated degradation of PD-L1, Mol. Cell 71 (2018) 606-620, https://doi.org/10.1016/j.molcel.2018.07. 030 e7.

[87] J. Zhang, X. Bu, H. Wang, Y. Zhu, Y. Geng, N.T. Nihira, et al., Cyclin D-CDK4 kinase destabilizes PD-L1 via cullin 3-SPOP to control cancer immune surveillance, Nature 553 (2018) 91-95, https://doi.org/10.1038/nature25015.

[88] P. D’Arrigo, M. Russo, A. Rea, M. Tufano, E. Guadagno, M.L. Del Basso De Caro, et al., A regulatory role for the co-chaperone FKBP51s in PD-L1 expression in glioma, Oncotarget 8 (2017) 68291-68304, https://doi.org/10.18632/oncotarget. 19309.

[89] S. Cuboni, S. Gaali, A. Kirschner, J. Hartmann, C. Kozany, G. Balsevich, C. Namendorf, P. Fernandez-Vizarra, C. Sippel, A.S. Zannas, R. Draenert, E.B. Binder, O.F. Almeida, G. Rühter, M. Uhr, M.V. Schmidt, C. Touma, A. Bracher, F. Hausch, Selective inhibitors of the FK506-binding protein 51 by induced fit, Nat. Chem. Biol. 11 (2015) 33-37, https://doi.org/10.1038/nchembio.1699.

[90] R.Q. Chen, F. Liu, X.Y. Qiu, X.Q. Chen, The prognostic and therapeutic value of PD-L1 in glioma, Front. Pharmacol. 9 (2019) 1503, https://doi.org/10.3389/ fphar.2018.01503.

[91] T. Azuma, S. Yao, G. Zhu, A.S. Flies, S.J. Flies, L. Chen, B7-H1 is a ubiquitous antiapoptotic receptor on cancer cells, Blood 111 (2008) 3635-3643, https://doi. org/10.1182/blood-2007-11-123141. 
[92] M. Ibañez-Vea, M. Gato-Cañas, M. Zuazo, H. Arasanz, L. Lorenzo, G. FernandezHinojal, et al., PDL1 signals through conserved sequence motifs to overcome interferon-mediated cytotoxicity, Cell Rep. 20 (2017) 1818-1829, https://doi.org/ 10.1016/j.celrep.2017.07.075.

[93] S. Chen, S. Liu, W. Yuan, H. Wang, K. Chen, D. Li, et al., PD-1/PD-L1 interaction up-regulates MDR1/P-gp expression in breast cancer cells via PI3K/AKT and MAPK/ERK pathways, Oncotarget 8 (2017) 99901-99912, https://doi.org/10 18632/oncotarget.21914.

[94] L. Zhao, C. Li, F. Liu, Y. Zhao, J. Liu, Y. Hua, et al., A blockade of PD-L1 produced antitumor and antimetastatic effects in an orthotopic mouse pancreatic cancer model via the PI3K/Akt/mTOR signaling pathway, Onco. Ther. 10 (2017) 2115-2126, https://doi.org/10.2147/OTT.S130481.

[95] Q. Zhang, Y. Zhang, Y. Chen, J. Qian, X. Zhang, K.A. Yu, Novel mTORC1/2 inhibitor (MTI-31) inhibits tumor growth, epithelial-mesenchymal transition, metastases, and improves antitumor immunity in preclinical models of lung cancer, Clin. Cancer Res. (2019), https://doi.org/10.1158/1078-0432.CCR-18-2548.

[96] X.Y. Qiu, D.X. Hu, W.Q. Chen, R.Q. Chen, S.R. Qian, C.Y. Li, et al., PD-L1 confers glioblastoma multiforme malignancy via Ras binding and Ras/Erk/EMT activation, Biochim. Biophys. Acta Mol. Basis Dis. 1864 (2018) 1754-1769, https://doi. org/10.1016/j.bbadis.2018.03.002.

[97] S. Xue, M. Hu, P. Li, J. Ma, L. Xie, F. Teng, et al., Relationship between expression of PD-L1 and tumor angiogenesis, proliferation, and invasion in glioma, Oncotarget 8 (2017) 49702-49712, https://doi.org/10.18632/oncotarget.17922.

[98] C.A. Clark, H.B. Gupta, G. Sareddy, S. Pandeswara, S. Lao, B. Yuan, et al., Tumorintrinsic PD-L1 signals regulate cell growth, pathogenesis, and autophagy in ovarian cancer and melanoma, Cancer Res. 76 (2016) 6964-6974, https://doi. org /10.1158/0008-5472.CAN-16-0258.

[99] S. Kraft, M.T. Fernandez-Figueras, N.A. Richarz, K.T. Flaherty, M.P. Hoang, PDL1 expression in desmoplastic melanoma is associated with tumor aggressiveness and progression, J. Am. Acad. Dermatol. 77 (2017) 534-542, https://doi.org/10. 1016/j.jaad.2017.05.007.

[100] L. Zhang, L.J. Xu, J. Zhu, J. Li, B.X. Xue, J. Gao, et al., ATM-JAK-PD-L1 signaling pathway inhibition decreases EMT and metastasis of androgenindependent prostate cancer, Mol. Med. Rep. 17 (2018) 7045-7054, https://doi.org/10.3892/mmr. 2018.8781.

[101] C. Zhang, F. Jiang, C. Su, P. Xie, L. Xu, Upregulation of long noncoding RNA SNHG20 promotes cell growth and metastasis in esophageal squamous cell carcinoma via modulating ATM-JAK-PD-L1 pathway, J. Cell. Biochem. (2019), https://doi.org/10.1002/jcb.28444.

[102] S. Kim, J. Koh, M.Y. Kim, D. Kwon, H. Go, Y.A. Kim, et al., PD-L1 expression is associated with epithelial-to-mesenchymal transition in adenocarcinoma of the lung, Hum. Pathol. 58 (2016) 7-14, https://doi.org/10.1016/j.humpath.2016.07. 007.

[103] S. Tsutsumi, H. Saeki, Y. Nakashima, S. Ito, E. Oki, M. Morita, et al., Programmed death-ligand 1 expression at tumor invasive front is associated with epithelial- mesenchymal transition and poor prognosis in esophageal squamous cell carcinoma, Cancer Sci. 108 (2017) 1119-1127, https://doi.org/10.1111/cas.13237.

[104] L. Chen, D.L. Gibbons, S. Goswami, M.A. Cortez, Y.H. Ahn, L.A. Byers, et al., Metastasis is regulated via microRNA-200/ZEB1 axis control of tumour cell PD-L1 expression and intratumoral immunosuppression, Nat. Commun. 5 (2014) 5241, https://doi.org/10.1038/ncomms6241.

[105] A. Alsuliman, D. Colak, O. Al-Harazi, H. Fitwi, A. Tulbah, T. Al-Tweigeri, et al., Bidirectional crosstalk between PD-L1 expression and epithelial to mesenchymal transition: significance in claudin-low breast cancer cells, Mol. Cancer 14 (2015) 149, https://doi.org/10.1186/s12943-015-0421-2.

[106] T. Maeda, M. Hiraki, C. Jin, H. Rajabi, A. Tagde, M. Alam, et al., MUC1-C induces PD-L1 and immune evasion in triple-negative breast cancer, Cancer Res. 78 (2018) 205-215, https://doi.org/10.1158/0008-5472.CAN-17-1636.

[107] H.B. Gupta, C.A. Clark, B. Yuan, G. Sareddy, S. Pandeswara, A.S. Padron, et al., Tumor cell-intrinsic PD-L1 promotes tumor-initiating cell generation and functions in melanoma and ovarian cancer, Signal Transduct. Target. Ther. 1 (2016), https://doi.org/10.1038/sigtrans.2016.30 pii: 16030.

[108] S. Almozyan, D. Colak, F. Mansour, A. Alaiya, O. Al-Harazi, A. Qattan, et al., PD-L1 promotes OCT4 and Nanog expression in breast cancer stem cells by sustaining PI3K/AKT pathway activation, Int. J. Cancer 141 (2017) 1402-1412, https://doi org/10.1002/ijc.30834.

[109] H. Wang, J. Deng, H.Y. Ren, P. Jia, W. Zhang, M.Q. Li, S.W. Li, Q.H. Zhou, STAT3 influences the characteristics of stem cells in cervical carcinoma, Oncol. Lett. 14 (2017) 2131-2136, https://doi.org/10.3892/ol.2017.6454.

[110] K. Tamai, M. Nakamura, M. Mizuma, M. Mochizuki, M. Yokoyama, H. Endo, et al., Suppressive expression of CD274 increases tumorigenesis and cancer stem cell phenotypes in cholangiocarcinoma, Cancer Sci. 105 (2014) 667-674, https://doi. org/10.1111/cas.12406.

[111] G.G. Jinesh, G.C. Manyam, C.O. Mmeje, K.A. Baggerly, A.M. Kamat, Surface PDL1, E-cadherin, CD24, and VEGFR2 as markers of epithelial cancer stem cells associated with rapid tumorigenesis, Sci. Rep. 7 (2017) 9602, https://doi.org/10. 1038/s41598-017-08796-z.

[112] S. Kleffel, C. Posch, S.R. Barthel, H. Mueller, C. Schlapbach, E. Guenova, et al., Melanoma cell-intrinsic PD-1 receptor functions promote tumor growth, Cell 162 (2015) 1242-1256, https://doi.org/10.1016/j.cell.2015.08.052.

[113] H. Li, X. Li, S. Liu, L. Guo, B. Zhang, J. Zhang, et al., Programmed cell death-1 (PD1) checkpoint blockade in combination with a mammalian target of rapamycin inhibitor restrains hepatocellular carcinoma growth induced by hepatoma cellintrinsic PD-1, Hepatology 66 (2017) 1920-1933, https://doi.org/10.1002/hep. 29360.

[114] J.L. Riley, PD-1 signaling in primary T cells, Immunol. Rev. 229 (2009) 114-125, https://doi.org/10.1111/j.1600-065X.2009.00767.x.

[115] H. Wang, H. Yao, C. Li, J.Y. Fang, J. Xu, Cancer cell-intrinsic PD-1 and implications in combinatorial immunotherapy, Front. Immunol. 9 (2018) 1774, https:// doi.org/10.3389/fimmu.2018.01774. 